\title{
A Haptic Tele-operated System for Microassembly
}

\author{
P. Estevez ${ }^{1}$, S. Khan ${ }^{1}$, P. Lambert ${ }^{1}$, M. Porta ${ }^{1}$, I. Polat ${ }^{2}$, \\ C. Scherer ${ }^{2}$, M. Tichem ${ }^{1}$, U. Staufer ${ }^{1}$, H.H. Langen ${ }^{1}$, and R. Munnig Schmidt ${ }^{1}$ \\ ${ }^{1}$ Precision and Microsystems Engineering, TU Delft \\ ${ }^{2}$ Delft Center for Systems and Control, TU Delft \\ Mekelweg 2 \\ 2628 CD Delft \\ The Netherlands \\ Tel.: +31 (0) 15 2786428; Fax: +31 (0) 152782150 \\ p.estevezcastillo@tudelft.nl
}

\begin{abstract}
A tele-haptic system for microassembly applications is currently being developed at the Delft University of Technology, with the goal of achieving superior performance by providing enhanced feedback to the human operator. Assembly of a micro-harmonic drive is used as a benchmark to fully evaluate the proposed tele-haptic system by investigating the control strategies and the individual subsystems: master device, microgrippers and slave system. The master device will be comprised of a parallel robot with a built-in gripper. The slave system and end effector are focused on providing efficient and effective force feedback of the interactions on the microenvironment to the human operator, in addition to detecting position and orientation of the object being grasped. Novel control strategies are also investigated to allow the transmission of high frequency transients to the operator, carrying information from hard contact interactions between the microgripper and the part to be assembled.
\end{abstract}

Keywords: Microassembly, Haptics, Teleoperation, Force Feedback, Parallel Robot, Event Based Haptics, Discretization.

\section{Introduction}

In today's emerging technologies, where the sizes of components are reaching the lower micrometer range, traditional automatic macro-assembly processes are being challenged to their limits. This is due to the requirements of high precision motion and small tolerances (usually less than a few micrometers). The predominance of surface forces over gravity complicates the release of parts, which tend to stick to the end-effector [1]. Furthermore, the handled parts are often delicate and fragile, requiring a control of forces in the micro-Newton range or below.

When dealing with microproducts that are produced in low-to-medium quantities with many variants, the automation of their assembly process may not be economically profitable because of the limited flexibility of the available assembly devices. For that reason the manual approach, often with the aid of a microscope, is 
presently the main method used in the assembly of low-to-medium series of microproducts. However, the pure manual approach is often not sufficient to fulfill the requirements, due to the difficulty of human operators to control the force and observe the precision aspects during the assembly.

Tele-operated systems [2], [3] are an interesting alternative to the unassisted manual assembly, but they fail on providing force feedback. Adding haptics to teleoperated systems becomes then a promising approach. The presence of the human operator, compared with the more rigid approach of automatic microassembly, improves the flexibility of the system thanks to his/her capability to plan, adapt, and react to unexpected situations during the assembly process. At the same time, the haptic-feedback reduces the risk of damaging handled parts and limits the assembly time, compared to regular tele-operated systems.

\section{State of the Art}

Tele-haptic systems have been used in different handling applications. Some researchers have utilized haptic interfaces in 1-DOF to perform basic operations like pushing [4] or more complicated functions for grasping micro-objects and carrying out assembly tasks [5], [6], [7]. Others have focused on biological applications by handling cells and tissue [8], [9]. Even if the results of using haptic technology for microassembly are promising, many challenges still need to be overcome with regard to design and control of multiple DOF haptic tele-operated systems for performing dexterous tasks. In particular there are different critical aspects that need research effort to improve the capabilities of the existing haptic environments:

i) The existing haptic environments present grasping force feedback, but no or little feedback in terms of interaction force (i.e. the force exerted on grasped microparts by other elements present in the assembly environment);

ii) Obstacles and the trade-off between high magnification and depth of field, result in difficulties in the manipulation when only visual feedback is used;

iii) Existing slave devices, such as [5] or [10], are not optimized for the presence of a human operator in the loop, resulting either in systems over-performing the user (and therefore unnecessarily expensive) or in non-transparent operations;

iv) Current commercial haptic masters, such as the well known Phantom or the Omega devices, are not specifically designed for 2.5 D microassembly tasks.

v) Device specific control schemes have trade-offs leading to high deficiencies in some performance aspects, and the passivity based design techniques [11] still remain the mainstream control algorithms.

\section{The Tele-haptic Environment and the Benchmark}

The development of a tele-haptic microassembly system is currently in progress at the Delft University of Technology (TUD), within MICRONED program [12].

First, and in order to choose a particular benchmark application, an analysis was made of assembly applications containing typical and relevant micro assembly tasks. 
This benchmark application is used to define the requirements for the master-slave robot combination and its tele-haptic environment. The analysis showed that many microassembly sequences consist of: pick-and-placing parts in relation to other parts, including defining a position in three translational degrees of freedom; aligning of parts around the vertical axis; and different kinds of peg-in-hole insertion, possibly requiring additional fine alignment on all $6 \mathrm{DOF}$. Some of the operations need fixing steps either by gluing, soldering, press fitting or releasing of flexible elements. Another important aspect pointed out by the analysis was that microproducts are often composed of fragile and delicate microparts and the risk of damaging them during the handling process is high. This is both due to high grasping forces, and environmental interaction forces on the manipulated parts. To avoid or at least reduce these risks, the control of the grasping and interaction force on the grasped microparts is of paramount importance.

A planetary and harmonic gearbox was chosen as benchmark application (Fig. 1) because its assembly process includes the typical assembly tasks described above. The assembly of this demonstrator-requires four DOF: three translations plus a rotation around the vertical $\mathrm{z}$ axis. The remaining rotations are expected to be less critical, and are planed to be addressed using the compliancy of the system and force sensing. Force feedback is required due to the fragility of the pegs, the low clearance, the slim gears and the need for part engaging. The parts of the demonstrator are going to be produced using MEMS technology, testing different shapes and dimensions.
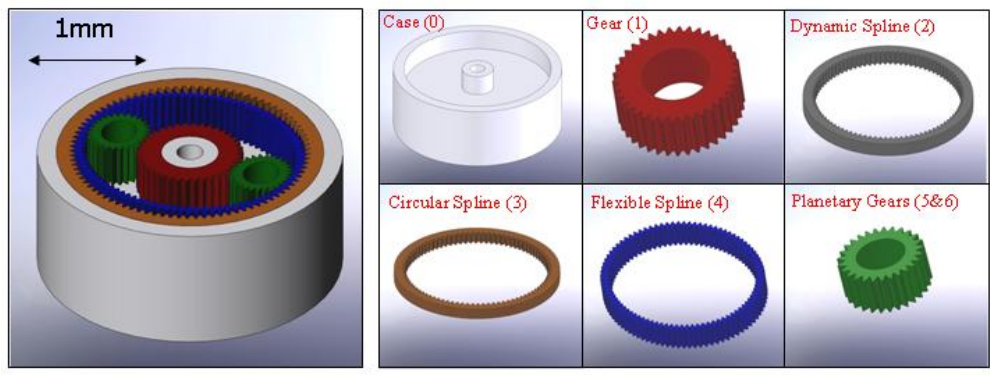

Fig. 1. The micro-harmonic drive benchmark and its components

As other tele-haptic systems, also this implementation is composed of a macroside, the master, which interfaces with the operator, and a micro-side, slave, operating in the environment in which the assembly tasks are actually carried out. The two sides are in mutual communication by a suitable control system that allows inputs and outputs to be transferred between the macro- and micro-side. Fig. 2a shows the structure of the macro-side: a parallel robot with four DOF is used as human interface. A gripper handle provides the gripping interaction in terms of grasping motion and interaction force feedback. Fig. 2b shows the micro-side of the environment: a positioning robot with three translational DOF is provided with the support for the microparts, and a mechanical two-finger microgripper is supported by a 1 DOF rotational stage. Suitable devices have to sense the grasping and environmental interaction force on the grasped object, and two cameras focus on the microgripper to 
monitor the assembly operations. Other end-effectors such as different kind of grippers, a glue dispenser, laser microwelding and UV-light sources can substitute the two finger mechanical gripper or can be mounted on independent supports within the micro-side of the system. For the moment these extensions are not considered. The remainder of this section discusses the main components of the system independently.

a)

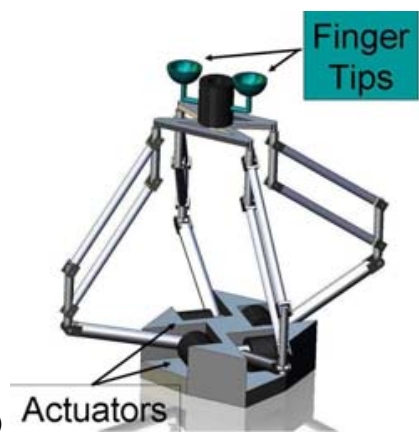

b)

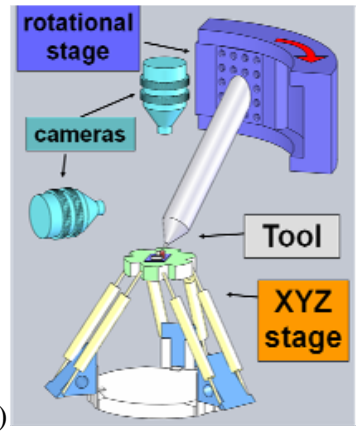

Fig. 2. The 4 DOF Master Robot and the 4 DOF Slave Robot with the gripper

\subsection{Macro-side of the Environment}

The role of the master robot is to provide interaction between the human operator and the rest of the system. The operator has to command a slave device and receive force feedback from the forces measured in the microenvironment. For realistic forcefeedback teleoperation, the human operator should feel only the forces that occur between the slave and the environment, and not the forces present in the master device itself. This means that the device has to show as little inertia as possible. On the other hand, to render high-frequency feedback from the microenvironment, the master device needs to have a high stiffness. The requirements of low inertia and high stiffness are contradictory and lead to a trade-off.

The chosen solution relies on a parallel robot architecture. Due to this architecture usually a stiffness/inertia ratio is achieved that is about one order of magnitude higher than regular serial robots. An innovative parallel mechanism generating the four DOF motion has been designed (Fig. 2a). The master device is equipped with two finger tips that represent the mechanical gripper. The center point between the two tips can be moved in the three translations and the tips can be rotated around the center point. Finally, it is possible to open and close the tips around the center point to command gripping actions.

Various analyses concerning the useful workspace, the stiffness, and the dexterity of the mechanism have been done. Due to the complexity of the mechanical system, most of the analyses and optimizations have been carried out using interval analysis [13], a powerful method that reduces the computing time and allows us to guarantee the algorithm results. An optimization of the various link dimensions was carried out to maximize the useful workspace of the robot. The useful workspace is a centered spherical region without singularities and with acceptable robot dexterity. For a robot height of $200 \mathrm{~mm}$, a preliminary optimization analysis gives us a $150 \mathrm{~mm}$ diameter 
sphere as useful workspace, where the gripping handle can be rotated \pm 65 degrees and the distance between the finger tips can vary from 40 to $80 \mathrm{~mm}$.

\subsection{Micro-side of the Environment}

The slave system must position the end effector relative to the parts support following the commands given by the operator. A work space of $20 \times 20 \times 10 \mathrm{~mm}^{3}$ must be covered, in order to reach all the micro-objects positioned on the microparts support, and sufficient resolution is needed to address assembly tasks with sub-micron clearances. The human actuation bandwidth of 3 to $10 \mathrm{~Hz}$ (depending on the kind of task) must also be matched. The requirements on drift and accuracy are less high, since the user is very capable of correcting for shortcomings in those aspects. Attention must be given to the possibility of mounting several end-effectors around the microassembly area, and to keep the area where the assembly is taking place always in focus. The presence of the human operator imposes additional constrains in order to obtain transparency and an intuitive operation.

The selected design, shown schematically in Fig. 2b, positions the parts support in three DOF, while the gripper rotates around the assembly point. By doing so, the vision system can stay focused on the microgripper/end effector, and the assembly area remains supervised regardless of the displacement of the microparts support. Furthermore, other tools can be mounted in the vicinity of the slave stage, and be used by positioning their end effectors into focus of the cameras. Adding a rotational degree of freedom to the base would improve the flexibility of the setup, but preliminary tests with a simulated environment suggest that it may be confusing for the human operator. Further psychophysical tests are been conducted to determine whether controlling all the degrees of freedom in the parts support, specially the rotational one, confuses the human operators affecting their performance, and to determine how to counteract this effect if this was the case. Additional psychophysical tests were conducted and consulted [14] to determine the average user capabilities for actuation and sensing, and map them into the slave workspace to ensure a transparent operation. Speeds of $400 \mathrm{~mm} / \mathrm{s}$ and accelerations of $4 \mathrm{~m} / \mathrm{s}$ were commonly observed in hand/wrist operation. Also, a range of $200 \mathrm{~mm}$ with resolution of $1 \mathrm{~mm}$ was identified (a 200:1 ratio), while the task covers a range of $20 \mathrm{~mm}$ with submicron resolution (a 100,000:1 ratio). Due to this difference, a multistep approximation to the goal will be necessary. In that way, for the roughest step a speed requisite of $40 \mathrm{~mm} / \mathrm{s}$ and acceleration of $400 \mathrm{~mm} / \mathrm{s}^{2}$ is set, together with a resolution of $100 \mu \mathrm{m}$; the last and finest step must give a resolution of $40 \mathrm{~nm}$ with speeds of 16 $\mu \mathrm{m} / \mathrm{s}$ and accelerations of $160 \mu \mathrm{m} / \mathrm{s}^{2}$, over a workspace of $8 \times 8 \times 8 \mu \mathrm{m}^{3}$. A parallel architecture is selected for the finest steps in order to minimize the moving mass, and flexure hinges are used to limit friction and provide high resolution motion.

The feedback architecture must provide two perpendicular views of the assembly area, force measurements from the gripping action, and force measurements from the interaction of the gripped part with the environment. Gripping forces should be high enough to hold, by friction, metallic and silicon based parts from tens to several hundreds of micrometers during transport and assembly, while avoiding damage to the slim gears and pegs. This yield to a requirement for sensing with a range up to $5 \mathrm{mN}$ and resolutions of micronewtons. Better resolutions would allow the user 
to control smaller interactions forces, protecting the parts and improving the positioning accuracy.

In order to provide the aforementioned visual feedback, two cameras will be located at a top and lateral position. For the environmental interaction force sensing two concepts are considered: inserting the force sensing device at wrist level (i.e. between the gripper and the support or between the base and the parts), or integrate interaction force sensors into the gripper itself. Solutions based on silicon microstructures with integrated sensors, strain gauges over metallic structures, or in measures of the positioning stage actuation power, are been compared in order to find the most appropriate principle. For the microgripper, silicon based grippers with integrated actuators and sensors are considered to be good solutions for satisfying the force requirements. The research has focused on the design of microgrippers with both grasping force and position sensing capability [15], [16]. The information about the object position within the gripper is usually detected by the visual feedback. The visual control however has intrinsic optical limitations such as its obstruction by obstacles on the workspace and the trade-off between depth of focus and magnification. Due to these visual control drawbacks, microgrippers that are able to detect the position and orientation of the object on the grasping surface can support and complement visual information, providing additional and important feedback. It can for instance detect whether the object is safely and correctly grasped or notice an unwanted movement of the object on the grasping surface. This information is of significant importance for successfully carrying out various assembly tasks [17]. Research efforts are still needed for providing these grippers with a suitable opening range to grasp micro objects of several sizes, up to a few hundreds of microns.

\subsection{Control}

The role of the controller is to match the position and force between the master and the slave system. 2-channels and 4-channels bilateral controllers have already been used in haptic teleoperation [18]. In haptic microassembly, the external forces acting on the work piece lead to position-dependent nonlinear characteristics. In order to avoid overly conservative control designs based on passivity techniques for ensuring stability and performances, it is of fundamental relevance to develop suitable structured uncertainty models (such as multivariable sector conditions on force nonlinearities) for the work-piece environment in haptic microassembly.

As a substantial benefit, these techniques even offer the opportunity to design controllers that adapt themselves to measurable changes of the environment in order to even further enhance performance. The current focus of the research is the hard contact stability problem, which is ubiquitous in the literature. The short-term goal is to characterize the stability properties of different control schemes, for instance [11], and develop a framework for robustness analysis specifically tailored to teleoperation.

Also, event-based haptic algorithms [19] are being researched because of their capability to present contact information to the user. It is for instance desirable for the user to acquire a rough idea about the stiffness of the material to be handled before performing any further procedures. Thus, research is being carried out to identify the material stiffness by means of tapping with the end-effector and generate corresponding signals for the operator. 


\section{Conclusion}

In this paper the tele-haptic environment in development at the Delft University of Technology is proposed as an alternative to the pure manual micro-assembly. The system will constitute a flexible and intuitive system for the assembly of microproducts in small and medium quantities. The analysis of existing microproducts led to the definition of a micro-harmonic drive as a benchmark suitable for microassembly systems and processes. This analysis also showed that actuation on four DOF is required for this system, and that the presence of grasping and interaction force feedback would enhance the performance over a wide variety of microassembly tasks. The particular characteristics of the suggested task and the involvement of a human operator, motivate the specifications for the environment, defining a new and interesting niche where the system will continue its development.

\section{Acknowledgment}

The authors wish to thank the technical staff of PME and the DIMES IC Process Group at TU Delft for their precious help in the development of the devices. Special thanks are due to Dr. Jia Wei for his cooperation in the research on microgrippers. This research is supported by the BSIK MicroNed program [12].

\section{References}

1. Fearing, R.S.: Survey of sticking effects for micro parts handling. In: IEEE/RSJ Int. Workshop on Intelligent Robots \& Systems (IROS 1995), Pittsburgh (1995)

2. Kunt, E.D.: Design and Realization of a Microassembly Workstation. MS Thesis, Sabanci University (2006)

3. Mitsuishi, M., Watanabe, T., Nakanishi, H., Hori, T., Watanabe, H., Kramer, B.: A telemicro-surgery system across the Internet with a fixed viewpoint/operation-point. In: IROS 1995, Pittsburgh, Pennsylvania, USA (August 1995)

4. Khan, S., Sabanovic, A.: Force Feedback Pushing Scheme for Micromanipulation Applications. Journal of Micro-Nano Mechatronics (accepted, 2009)

5. Beyeler, F., Probst, M., Nelson, B.J.: A Microassembly System with Microfabricated Endeffectors for Automated Assembly Tasks. In: Workshop on Robotic Microassembly of 3D Hybrid MEMS, IEEE/RSJ Int. Conf. on Intelligent Robots and Systems (October 2007)

6. Zhou, Q., Aurelian, A., del Corral, C., Esteban, P.J., Kallio, P., Chang, B., Koivo, H.N.: Microassembly station with controlled environment. In: Proc. of SPIE, vol. 4568, pp. 252 $260(2001)$

7. Hériban, D., Agnus, J., Gauthier, M.: Micro-manipulation of silicate micro-sized particles for biological applications. In: 5th Int. Workshop on Microfactories, Besançon, France (2006)

8. Sieber, A., Valdastri, P., Houston, K., Eder, C., Tonet, O., Menciassi, A., Dario, P.: A novel haptic platform for real time bilateral biomanipulation with a MEMS sensor for triaxial force feedback. Sensors and Actuators A 142, 19-27 (2008) 
9. Arai, F., Sugiyama, T., Luangjarmekorn, P., Kawaji, A., Fukuda, T., Itoigawa, K., Maeda, A.: 3D viewpoint selection and bilateral control forbio-micromanipulation. In: IEEE Int. Conf. on Robotics and Automation (ICRA 2000), vol. 1, pp. 947-952 (2000)

10. Perroud, S., Codourey, A., Mussard, Y.: PocketDelta: a miniature robot for microassembly. In: 5th International Workshop on MicroFactories, Besançon, France (October 2006)

11. Colgate, J.E., Schenkel, G.G.: Passivity of a Class of Sampled-Data Systems: Application to Haptic Interfaces. Journal of Robotic Systems 14(1), 37-47 (1997)

12. Microned Programme, http: / /www. microned.nl/

13. Moore, R.E., Bierbaum, F.: Methods and Applications of Interval Analysis. Society for Industrial Mathematics (1979)

14. Tan, H.Z., Srinivasan, M.A., Eberman, B., Cheng, B.: Human factors for the design of force-reflecting haptic interfaces. In: 3rd Int. Symposium on Haptic Interfaces for Virtual Environment and Teleoperator Systems, Chicago, IL, vol. 55/1, pp. 353-359 (1994)

15. Porta, M., Wei, J., Tichem, M., Sarro, P.M., Staufer, U.: Vertical Contact Position Detection and Grasping Force Monitoring for Micro-Gripper Applications. In: IEEE Sensors 2009 (2009)

16. Wei, J., Porta, M., Tichem, M., Sarro, P.M.: A Contact Position Detection and Interaction Force Monitoring Sensor for Micro-Assembly Applications. In: Transducers 2009, Denver, CO, USA, pp. 2385-2388 (2009)

17. Porta, M., Tichem, M.: Grasping and Interaction Force Feed-Back in Microassembly. In: Accepted to the 5th Int. Prec. Assembly Seminar (IPAS 2010), Chamonix, France, February 14-17 (2010)

18. Hannaford, B.: A design framework for teleoperators with kinesthetic feedback. IEEE Transactions on Robotics and Automation 5(4), 426-434 (1989)

19. Kuchenbecker, K.J., Fiene, J., Niemeyer, G.: Improving Contact Realism Through EventBased Haptic Feedback. IEEE Transactions on Visualization and Computer Graphics 2(2), 219-230 (2006) 